\title{
Família e escola: Perspetivas sobre a utilização de meios tecnológicos e
}

\section{segurança}

\section{Family and school: Perspectives on the use of technology and security}

\author{
Ana Margarida Veiga Simão , Paula Paulino ${ }^{*}$, Paula Costa Ferreira ${ }^{*}$, Susana Costa Ramalho ${ }^{*}$, Sofia Francisco ${ }^{*}$, \\ Sidclay Bezerra de Souza* \\ *Universidade de Lisboa, Portugal, ${ }^{* *}$ Universidade Lusófona de Lisboa.
}

\begin{abstract}
Resumo
O presente estudo teve como objetivo compreender como os jovens reportam a utilização de meios tecnológicos e conhecer a perceção que encarregados de educação e professores têm acerca dessa utilização. Propôs-se ainda perceber se estes monitorizam a utilização que os jovens fazem dos meios tecnológicos e que práticas de sensibilização utilizam. Foram aplicados o Inventário de Incidentes Observados de Cyberbullying a 269 adolescentes e o Inventário sobre Segurança Online e Cyberbullying a 155 encarregados de educação e 28 professores. Evidenciam-se diferenças significativas entre a utilização reportada pelos alunos e as perceções dos encarregados de educação e professores.

Palavras-chave: [Jovens, Encarregados de Educação, Professores, Tecnologia, Segurança.]
\end{abstract}

\begin{abstract}
This study aimed to understand how adolescents report the use of technological resources and to know the perception guardians and teachers have about this use. The study proposed to provide an understanding of whether these adults monitor adolescents' use of technological resources, as well as identify practices of sensitization. The Inventory of Observed Incidents of Cyberbullying was applied to 269 adolescents and the Online Safety and Cyberbullying Inventory to 155 guardians and 28 teachers. Significant differences were found between the reported Internet use by the students and the perceptions of parents and teachers regarding that use.

Keywords: [Youth, Parents, Teachers, Technology, Safety.]
\end{abstract}

O avanço constante da tecnologia e a crescente utilização feita pelos adolescentes de meios tecnológicos constituem aspetos importantes para a interação social e comunicação online (Rivers \& Noret, 2010). Estes fenómenos acarretam potenciais riscos, como por exemplos a violação de privacidade e o cyberbullying (Moreno, Egan, Bare, Young, \& Cox, 2013). É neste âmbito que surgiu o projeto Cyberbullying: A regulação do comportamento através da linguagem (PTDC/MHC-PED/3297/2014) e que pretende investigar os comportamentos de cyberbullying dos adolescentes, bem como a linguagem que utilizam nestas interações, com vista à criação de uma aplicação para a prevenção e intervenção neste fenómeno. Assumindo que uma utilização segura da Internet pode ser desenvolvida através da formação educativa, acredita-se que o papel dos encarregados de educação, dos pais e dos próprios adolescentes, se torna fundamental para a promoção de comportamentos pró-sociais online. Assim, numa primeira etapa deste projeto, procurou-se compreender como os jovens reportam a utilização de meios tecnológicos e conhecer a perceção que encarregados de educação e professores têm acerca dessa utilização. Pretendeu-se de igual forma, perceber se estes monitorizam a utilização que os jovens fazem dos meios tecnológicos e que práticas de sensibilização utilizam quanto aos eventuais riscos e as questões de segurança no uso da Internet.

De um modo geral, a literatura atual tem revelado que a comunicação online apresenta efeitos sobre o desenvolvimento psicossocial dos adolescentes (e.g., Rivers \& Noret, 2010). Alguns estudos referem que a possibilidade de uma maior interação social constitui um benefício da utilização das tecnologias, que se associa ao bem-estar dos adolescentes (e.g., Valkenburg \& Peter, 2011). Em contrapartida, outras investigações revelam que a forma como os adolescentes utilizam a Internet e que os recursos tecnológicos acabam por apresentar consequências negativas ao longo do desenvolvimento dos mesmos (e.g., Wright, 2017). Em termos de riscos, importa destacar os comportamentos aditivos em relação ao uso da Internet (Park, Kim, \& Cho, 2008) e ainda o envolvimento em situações de cyberbullying (Veiga Simão et al., 2017). Numa investigação conduzida por Navarro, Serna, Martínez e Ruiz-Oliva (2013), os resultados indicam que o uso da Internet, especificamente a comunicação online, aumenta a probabilidade de vitimização do cyberbullying.

Num estudo desenvolvido por Livingstone, Ólafsson e Staksrud (2011) verificou-se que 78\% dos adolescents em Portugal com idades entre os 13 a 16 anos utilizam a Internet. De acordo com Sozio et al (2015), esta utilização acontece maioritariamente em casa (86\%) e, em seguida, no contexto escolar (49\%). No que diz 
respeito aos dispositivos com que acedem à Internet, o estudo revela que $79 \%$ dos estudantes utilizam o portátil para aceder à Internet e $61 \%$ utilizam o smartphone (Sozio, et al., 2015). Estes resultados são importantes pois revelam uma elevada percentagem na utilização da Internet e que o uso que os adolescentes fazem ocorre, concomitantemente, em diferentes contextos e dispositivos.

Relativamente ao cyberbullying, um estudo recente de Veiga Simão et al. (2017) revelou que $15 \%$ dos estudantes foram vítimas de cyberbullying. Estes resultados devem ter em consideração a tendência dos implicados no fenómeno tenderem a subestimar o seu envolvimento no mesmo (Francisco, Veiga Simão, Ferreira, \& Martins, 2015).

A literatura tem evidenciado que os encarregados de educação são considerados pelos professores e profissionais educativos (e pelos próprios) como sendo os principais responsáveis pela formação dos educandos relativamente à utilização da Internet (Moreno et al., 2013). Por conseguinte, torna-se impreterívelque os encarregados de educação tenham conhecimento da atividade online dos seus educandos (Ang, Chong, Chye, \& Huan, 2012). Isto porque estudos recentes têm evidenciado que quando existe uma monitorização por parte dos encarregados de educação na utilização que os adolescentes fazem da Internet, a probabilidade de incorrerem em problemas relacionados com esta utilização, como por exemplo, agressões online, é menor (Kalmus, Blinka, \& Ólafsson, 2015). Além disso, outros estudos (e.g., Dehue, Bolman, \& Völlink, 2008) evidenciam que a maioria dos pais estabelece regras para os seus filhos sobre o uso da Internet, mas não estão efetivamente conscientes dos potenciais incidentes. Eles subestimam, por exemplo, o comportamento de cyberbullying dos seus próprios filhos e têm uma noção insuficiente dos mesmos como potenciais vítimas de cyberbullying (Dehue et al., 2008). A título de exemplo, a monitorização por parte dos pais, a criação conjunta de regras relativas ao tempo de uso da Internet e às informações pessoais compartilhadas, ajudam a diminuir a probabilidade de vitimização online (Navarro et al., 2013). De acordo com Mesch (2009), algumas técnicas de mediação usadas pelos pais têm um efeito protetor (e.g., mediação avaliativa sobre o acesso a determinados sítios online).

Relativamente ao papel dos professores, os estudos referem que as perceções destes profissionais devem ser tidas em consideração na elaboração de programas de prevenção e intervenção no que diz respeito à má utilização da Internet, como por exemplo, situações de cyberbullying (Stauffer, Heath, Coyne, \& Ferrin, 2012). Em ambientes escolares (contextos onde os adolescentes passam grande parte do seu tempo) os professores são responsáveis pela monitorização das atividades realizadas pelos seus alunos, pelo reforço de comportamentos positivos e pela imposição de consequências disciplinares a comportamentos inapropriados (Stauffer et al., 2012). Nos últimos anos, tem-se assistido a um aumento de novos comportamentos online que constituem desafios a esta gestão comportamental (Hinduja \& Patchin, 2010).
Além disso, os professores representam elementos fundamentais no apoio a intervenções que promovem o desenvolvimento emocional e escolar dos alunos (e.g., Forman, Olin, Hoagwood, Crowe, \& Saka, 2009). Neste sentido, as perceções destes profissionais relativamente à utilização que os seus alunos fazem da Internet, e especialmente, aos perigos relacionados com esta utilização, devem ser considerados para o delineamento de programas educativos em contextos escolares (Stauffer et al., 2012). Mais ainda, e de acordo com alguns autores, é fundamental dar formação aos professores para que estes tenham uma maior integração nos programas educativos implementados nas suas escolas no âmbito da utilização segura da Internet (Hirschstein, Edstrom, Frey, Snell, \& McKenzie, 2007).

Neste sentido, considerando-se os diversos riscos que podem advir da utilização da Internet e dos dispositivos tecnológicos, o envolvimento da família e da escola apresenta-se como fundamental (Souza, Veiga Simão, \& Caetano, 2014).

\section{Método}

\section{Participantes}

Participaram um total de 269 alunos (42.4\% do género feminino e $57.6 \%$ do género masculino), do $5 .^{\circ}$ ao $12 .^{\circ}$ ano de escolaridade com idade entre os 10 e 19 ou mais anos. Participaram ainda 155 encarregados de educação, $7,1 \%$ dos quais mencionaram ter pouca ou nenhuma experiência na utilização da Internet, $38,1 \%$ referiram ter alguma experiência na utilização da Internet e por fim, 54,8\% reportaram ter bastante ou muita experiência com a Internet. Relativamente aos 28 professores que participaram no estudo, $7,1 \%$ mencionaram ter pouca experiência na utilização da Internet, $50 \%$ referiram ter alguma experiência e $42,9 \%$ reportaram ter bastante ou muita experiência com a Internet.

\section{Instrumentos}

Foi utilizado o Inventário de Incidentes Observados de Cyberbullying (IOOC), desenvolvido por Ferreira, Veiga Simão, Paulino, Souza e Francisco (2017) de acordo com o Modelo de Intervenção do Observador (Darley \& Latané, 1968) e adaptado a partir da escala dos observadores do Questionário de Cyberbullying no Ensino Superior (Francisco et al., 2015). Também foi utilizado o Inventário sobre Segurança Online e Cyberbullying (ISOC), sendo uma versão para os Encarregados de Educação e outra para Professores. Estes instrumentos foram desenvolvidos pela equipa de investigadores do Programa de Estudos sobre o Cyberbullying (PEC) no âmbito de dois projetos de investigação financiados pela Fundação para a Ciência e Tecnologia (FCT): "Cyberbullying: The regulation of behavior through language." (PTDC/MHCPED/3297/2014) e "The Bystander Effect in Cyberbullying - taking responsibility and intervening through the regulation of behavior in adolescence." (SFRH/BPD/110695/2015). 


\section{Procedimento de recolha e análise dos dados}

A aplicação dos instrumentos decorreu após a obtenção de autorização por parte da Proteção Nacional de Dados, do Ministério da Educação, da Comissão de Ética e Deontologia da Faculdade de Psicologia da Universidade de Lisboa e do Diretor do Agrupamento Escolar. Foi também solicitada a colaboração dos participantes e seus respetivos encarregados de educação e professores e fornecidas informações relativamente às questões éticas implicadas na investigação (e.g., anonimato, apoio psicológico em caso de necessidade, etc.).

O IOOC foi aplicado em contexto de sala de aula, ao passo que as versões do ISOC foram enviadas através de email aos encarregados de educação e aos professores. Todos os instrumentos foram respondidos através da plataforma Qualtrics. Todo o procedimento de recolha envolveu um agrupamento escolar situado na região de Lisboa. Atendendo aos objetivos do estudo, os dados foram analisados através do software IBMSPSS 23.0 .

\section{Resultados}

De acordo com os objetivos definidos, os resultados obtidos serão apresentados segundo três dimensões, mais especificamente: utilização dos meios tecnológicos; a perceção que os encarregados de educação e os professores têm acerca dessa utilização; de que forma os encarregados de educação monitorizam a utilização que os estudantes fazem das plataformas e/ou redes sociais e a perceção que os professores têm dessa monitorização.

\section{Utilização das tecnologias pelos adolescentes}

Sobre a utilização das tecnologias, destacam-se como as mais utilizadas pelos estudantes: Telemóvel (97.0\%); Computador (91.1\%); Tablet (73.4\%); e Consolas (68.8\%). No que concerne à frequência com que os adolescentes acedem às plataformas e/ou redes sociais, evidencia-se a utilização de mais que uma vez por dia do Youtube (65.8\%), Instagram (59.9\%), Whatsapp (46.5\%), o SnapChat (41.6\%), como também o Facebook (32.3\%). Por outro lado, o Tumblr (66.5\%) e o Twitter (53.5\%), são as plataformas e/ou redes sociais que os adolescentes reportam nunca terem acedido, como apresentado na Tabela 1 .
Tabela 1.

Frequência com que os adolescentes acedem às plataformas elou redes sociais

\begin{tabular}{lcccccc}
\hline Plataformas & 1 & 2 & 3 & 4 & 5 & 6 \\
\hline Twitter & 53.5 & 9.3 & 7.1 & 6.3 & 9.3 & 14.5 \\
Facebook & 11.9 & 2.6 & 9.7 & 19.0 & 24.5 & 32.3 \\
Whatsapp & 14.9 & 3.7 & 7.8 & 9.3 & 17.8 & 46.5 \\
Google+ & 30.5 & 17.8 & 15.6 & 14.1 & 8.9 & 13.0 \\
Youtube & 0.4 & 1.1 & 1.5 & 11.9 & 19.3 & 65.8 \\
Tumblr & 66.5 & 13.8 & 6.7 & 4.8 & 2.2 & 5.9 \\
Instagram & 20.1 & 2.2 & 1.5 & 4.5 & 11.9 & 59.9 \\
SnapChat & 24.2 & 4.1 & 6.7 & 7.8 & 15.6 & 41.6 \\
Skype & 17.1 & 19.0 & 28.3 & 19.3 & 10.3 & 6.3 \\
Emails & 5.6 & 2.6 & 16.0 & 32.7 & 26.8 & 16.4 \\
\hline
\end{tabular}

Nota: $1=$ Nunca acedi; $2=$ Uma vez por ano; $3=$ Uma vez por mês; $4=$ Uma vez por semana; $5=$ Uma vez por dia; $6=$ Mais que uma vez por dia; os valores apresentados nesta tabela tratam-se de percentagens

Relativamente a quem inscreveu os adolescentes nas plataformas e/ou redes sociais acima referidas, os participantes referiram ter sido o próprio $(82.5 \%)$, os pais $(13.0 \%)$, um amigo (3.3\%), outro familiar $(0.7 \%)$, e apenas $0.4 \%$ responderam como não aplicável. Em relação à idade do primeiro acesso, as maiores percentagens centraram-se entre os 9 e 13 anos, conforme apresentado na Figura 1.

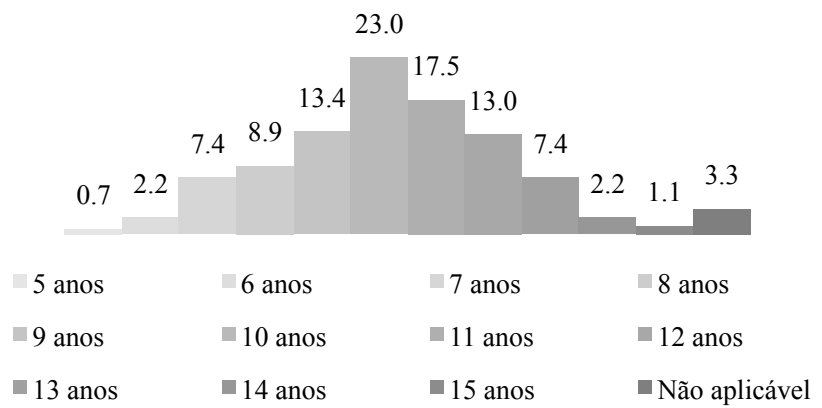

Figura 1. Idade do primeiro acesso a plataformas e/ou redes sociais

No que diz respeito ao tempo de utilização diária da Internet, excluindo as tarefas escolares, os resultados são apresentados na Tabela 2.

Tabela 2.

Tempo de utilização diária da Internet por parte dos adolescentes

\begin{tabular}{lc}
\hline Tempo diário & Frequência \\
\hline 30 minutos & 7.8 \\
1 a 2 horas & 34.6 \\
3 e 4 horas & 37.5 \\
5 e 6 horas & 11.5 \\
Mais que 6 horas & 8.6 \\
\hline
\end{tabular}


Nota: os valores apresentados nesta tabela são referentes a percentagens

Quanto ao conteúdo do que é partilhado na Internet pelos adolescentes, verificamos que $4.1 \%$ partilham fotos de outras pessoas, $3.7 \%$ partilham fotos pessoais e vídeos de outras pessoas e $3.3 \%$ publicam informações gerais (e.g., campanhas, notícias, eventos, etc.) "mais que uma vez por dia".

\section{Perspetivas de encarregados de educação e professores sobre a utilização dos recursos tecnológicos}

Os dados deste estudo evidenciam diferenças significativas entre a utilização/frequência de utilização das plataformas digitais reportada pelos alunos e as perceções dos encarregados de educação e professores acerca da mesma. Especificamente, os professores percecionaram uma maior frequência de utilização do que a reportada pelos alunos e mencionada pelos encarregados de educação, e.g., Twitter, $[F(2,449)=65$, $51, p<0.01]$.

\section{Monitorização e práticas de sensibilização por parte dos encarregados de educação e dos professores}

Em termos de práticas de segurança, a maioria dos encarregados de educação mencionou a existência de regras de restrição em casa $(63.2 \%)$ quanto ao tempo de utilização da Internet e a não existência dessas regras relativamente $(65.8 \%)$ ao espaço físico de acesso e (66.5\%) aos sítios visitados. A maioria dos professores percecionou a existência de regras de restrição em casa (75\%) quanto ao tempo de utilização da Internet e redes sociais, (67.9\%) ao espaço físico de acesso, e (53.6\%) aos sítios visitados apenas para alguns alunos. A maioria dos encarregados de educação mencionou ainda a frequente monitorização do uso da Internet dos educandos $(44.5 \%)$, bem como o conhecimento das palavras-passe dos últimos (45.2\%).

Relativamente às práticas de sensibilização quanto aos eventuais riscos e as questões de segurança no uso da Internet, a maioria dos encarregados de educação mencionou discutir com os educandos questões relacionadas com o uso da Internet frequentemente $(66.5 \%)$, enquanto a maioria dos professores referiu discutir às vezes $(46.4 \%)$.

\section{Discussão}

Os resultados deste estudo constituem um importante contributo acerca da utilização que as crianças e adolescentes em Portugal fazem dos recursos tecnológicos de que dispõem, da perceção que os encarregados de educação e os professores têm acerca dessa utilização, da monitorização por parte dos encarregados de educação e as perceções dos professores sobre essa monitorização e a utilização que os adolescentes fazem das plataformas e/ou redes sociais.

Concluiu-se que existem discrepâncias entre o que os alunos reportam e a perceção dos professores relativamente à utilização dos meios tecnológicos. Ainda, os encarregados de educação evidenciaram uma maior frequência de práticas de sensibilização do que os professores, e menor implementação de regras de segurança do que as percecionadas pelos últimos.

Os resultados obtidos acerca dos meios pelos quais os estudantes utilizam a Internet são semelhantes ao estudo de Sozio et al (2015) pois indicam que os computadores e os telemóveis são os meios que apresentam maior frequência no acesso à Internet por parte dos adolescentes. Adicionalmente, este estudo permitiu perceber que as plataformas e/ou redes sociais que são mais utilizadas pelos adolescentes são o Youtube (65.8\%), Instagram (59.9\%), Whatsapp (46.5\%), o SnapChat (41.6\%), como também o Facebook (32.3\%).

Destacamos neste sentido, a relevância da monitorização por parte da família e da escola, como ainda sobre a importância da existência de regras acerca da utilização dos meios tecnológicos, de um modo geral, uma vez que os estudantes não estão isentos dos possíveis riscos como o comportamento aditivo em relação à Internet (Park et al, 2008), como ainda de outros tipos de situações que podem provocar diversos danos à saúde e ao desenvolvimento biopsicossocial dos adolescentes, como por exemplo o envolvimento em situações de cyberbullying (Veiga Simão et al., 2017).

Considerando que os efeitos nocivos das experiências negativas online são consideravelmente reduzidas pela oportunidade de discutir esta temática com os pais (e.g. Matsunaga, 2011), importa que estes se sintam preparados para abordar o tema e capazes de intervir eficazmente na sua prevenção.

Adicionalmente, os resultados vão ao encontro do estudo de Stauffer e colegas (2012), em que os professores identificaram o envolvimento dos pais como a estratégia mais eficaz para diminuir as experiências negativas online, como o cyberbullying. Por outro lado, diversos estudos têm salientado que os alunos são mais propensos a situações de cyberbullying quando há uma falta de monitorização parental das atividades online (Willard, 2005).

As tecnologias da informação e comunicação criaram novos desafios para os educadores, que se percecionam como não preparados e um tanto incertos sobre como lidar com esta realidade (Shariff, 2011). Neste sentido, enfatiza-se a relevância da continuidade de investigações que sejam capazes de orientar futuras intervenções que promovam o comportamento pró-social a partir da própria tecnologia com o conhecimento dos pais e dos professores. Ou seja, a tecnologia pode ser utilizada para fomentar a criação de redes sociais saudáveis e geradoras de novas e múltiplas interações entre os adolescentes. Além disso, este estudo procurou dar ênfase ao papel que a família e a escola desempenham no desenvolvimento e comportamento das crianças e dos adolescentes (Navarro et al., 2013). Estes intervenientes podem assumir este papel a partir do momento em que são capazes de identificar o tipo de relações que os adolescentes estabelecem no seu quotidiano (Mesch, 2009; Vieira, Mendes, \& Guimarães, 2009) e estejam efetivamente conscientes dos desafios, da importância da implementação de regras de segurança e das 
potencialidades que a tecnologia apresenta em termos do desenvolvimento psicossocial dos últimos.

\section{Conclusão}

As diferenças encontradas nas perceções dos encarregados de educação e dos professores acerca da utilização da Internet por parte dos adolescentes e relativamente às práticas de sensibilização e monitorização sugerem a necessidade de promover a formação ao nível da comunidade educativa. Adicionalmente, o conhecimento acerca das perceções sobre a utilização da Internet, bem como das práticas de sensibilização e monitorização, constituem variáveis do contexto a ter em conta no desenvolvimento de uma aplicação digital que promove o comportamento pró-social no âmbito do projeto Cyberbullying: $A$ regulação do comportamento através da linguagem.

\section{Referências}

Ang, R., Chong, W., Chye, S., \& Huan, V. (2012). Loneliness and generalized problematic Internet use: Parents' Perceived knowledge of adolescents' online activities as a moderator. Computers in Human Behavior, 28, 1342-1347. doi: 10.1016/j.chb.2012.02.019.

Darley, J., \& Latane, B. (1968) Bystander intervention in emergencies: diffusion of responsibility. Journal of Personality and Social Psychology, 8(4), 377-383.

DeHue, F., Bolman, C., \& Völlink, T. (2008). Cyberbullying: Youngsters' experiences and parental perception. CyberPsychology \& Behavior, 11(2), 217-223

Ferreira, P., Veiga Simão, A.M., Paulino, P., Souza, S. B., \& Francisco, S. M. (2017, Julho). Assessing Bystander Behavior in Incidents of Cyberbullying. Oral communication no 14th European Conference on Psychological Assessment realizado no ISCTE Instituto Universitário de Lisboa, Lisboa, Portugal.

Forman, S. G., Olin, S. S., Hoagwood, K. E., Crowe, M., \& Saka, N. (2009). Evidence-based interventions in schools: Developers' views of implementation barriers and facilitators. School Mental Health, 1(1), $26-36$.

Francisco, S. M., Veiga Simão, A. M. V., Ferreira, P. C., \& Martins, M. J. D. (2015). Cyberbullying: The hidden side of college students. Computers in Human Behavior, 43, 167-182.

Hinduja, S., \& Patchin, J. W. (2010). Bullying, cyberbullying, and suicide. Archives of Suicide Research, 14, 206 - 221.

Hirschstein, M. K., Edstrom, L.V.S., Frey, K. S., Snell, J. L., \& MacKenzie, E. P. (2007). Walking the talk in bullying prevention: Teacher implementation variables related to initial impact of the Steps to Respect program. School Psychology Review, 36(1), $3-21$.

Kalmus, V., Blinka, L., \& Ólafsson, K. (2015). Does it matter what mama says: Evaluating the role of parental mediation in European Adolescents' Excessive Internet use. Children \& Society, 29, 122-133. doi: $10.1111 /$ chso. 12020
Livingstone, S., Ólafsson, K., \& Staksrud, E. (2011). Social networking, age and privacy. LSE London: EU Kids Online.

Matsunaga, M. (2011). Underlying circuits of social support for bullied victims: An appraisal-based perspective on supportive communication and postbullying adjustment. Human Communication Research, 37, 174-206.

Moreno, M., Egan, K., Bare, K., Young, H., \& Cox, E. (2013). Internet safety education for youth: stakeholder perspectives. BMC Public Health, 13(543). doi: 10.1186/1471-2458-13-543.

Mesch, G. S. (2009). Parental mediation, online activities, and cyberbullying. CyberPsychology \& Behavior, 12(4), 387-393.

Navarro, R., Serna, C., Martínez, V., \& Ruiz-Oliva, R. (2013). The role of Internet use and parental mediation on cyberbullying victimization among Spanish children from rural public schools. European journal of psychology of education, 28(3), 725-745.

Park, S. K., Kim, J. Y., \& Cho, C. B. (2008). Prevalence of Internet addiction and correlations with family factors Among South Korean adolescents. Adolescence, 43(172), 895 - 909.

Rivers, I., \& Noret, N. (2010). 'I h8 u': findings from a five-year study of text and email bullying. British Educational Research Journal, 36(4), 643-671. doi: 10.1080/01411920903071918.

Shariff, S. (2011). Cyberbullying: Questões e soluções para a escola, a sala de aula e a família. Porto Alegre, RS: Artmed.

Sozio, M. E., Ponte, C., Sampaio, I. V., Senne, F., Ólafsson, K., Alves, S. J., ... \& Mascheroni, G. (2015). Children and Internet use: A comparative analysis of Brazil and seven European countries. London: London School of Economics, EU Kids Online.

Souza, S. B., Simão, A. M. V., \& Caetano, A. P. (2014). Cyberbullying: Percepções acerca do Fenômeno e das Estratégias de Enfrentamento. Psicologia: Reflexão e Crítica, 27(3), 582-590.

Stauffer, S., Heath, M. A., Coyne, S. M., \& Ferrin, S. (2012). High school teachers' perceptions of cyberbullying prevention and intervention strategies. Psychology in the Schools, 49(4), 352-367.

Valkenburg, P., \& Peter, J. (2011). Online Communication Among Adolescents: An Integrated Model of Its Attraction, Opportunities, and Risks. Journal of Adolescent Health 48(2), 121-7. doi: 10.1016/j.jadohealth.2010.08.020.

Veiga Simão, A. M., Ferreira, P. C., Freire, I., Caetano, A. P., Martins, M. J., \& Vieira, C. (2017). Adolescent cybervictimization - Who they turn to and their perceived school climate. Journal of Adolescence, 58, 12-23.

Vieira, T. M., Mendes, F. D. C., \& Guimarães, L. C. (2009). De Columbine à Virgínia Tech: Reflexões com base empírica sobre um fenômeno em expansão. Psicologia: Reflexão e Crítica, 22(3), 493-501.

Willard, N. (2005). Educator's guide to cyberbullying addressing the harm caused by online social cruelty. Retrieved July, 19, 2005. 
Wright, M. F. (2017). The Role of Technologies, Behaviors, Gender, and Gender Stereotype Traits in Adolescents' Cyber Aggression. Journal of Interpersonal Violence, 1-20.

\section{Agradecimentos}

Agradecemos a Fundação para Ciência e Tecnologia
(FTC) pelos
projetos
financiados

(PTDC/MHC-PED/3297/2014;

SFRH/BPD/110695/2015). 